\title{
Low impact of staging EUS for determining surgical resectability in esophageal cancer
}

\author{
M. van Zoonen • M. G. H. van Oijen • \\ M. S. van Leeuwen $\cdot$ R. van Hillegersberg • \\ P. D. Siersema $\cdot$ F. P. Vleggaar
}

Received: 18 December 2011/ Accepted: 19 March 2012/Published online: 13 June 2012

(C) The Author(s) 2012. This article is published with open access at Springerlink.com

\begin{abstract}
Background Although studies have shown that EUS has a high sensitivity and specificity for $\mathrm{T}$ and $\mathrm{N}$ staging, the value of EUS for staging tumors as resectable or nonresectable after CT of the chest and abdomen and US neck assessment, is largely unknown. This study was designed to assess the diagnostic value of EUS for determining resectability of esophageal cancer.

Methods A retrospective analysis of all consecutive patients with esophageal carcinoma who underwent staging EUS, CT, and US. Tumors were considered resectable when there was no evidence of metastases or ingrowth in adjacent structures. Sensitivity, specificity, positive (PPV), and negative (NPV) predictive value of CT/US neck and CT/US neck + EUS for predicting surgical resectability were calculated. PPVs of CT/US alone and CT/US + EUS
\end{abstract}

M. van Zoonen · M. G. H. van Oijen · P. D. Siersema •

F. P. Vleggaar ( $\square)$

Department of Gastroenterology and Hepatology, University

Medical Center Utrecht, P.O. Box 85500, 3508 GA Utrecht,

The Netherlands

e-mail: f.vleggaar@umcutrecht.nl

M. van Zoonen

e-mail: margreetvanzoonen@gmail.com

M. van Zoonen · M. G. H. van Oijen · P. D. Siersema ·

F. P. Vleggaar

University Medical Center Utrecht, 3508 Heidelberglaan 100,

3584 CX Utrecht, The Netherlands

M. S. van Leeuwen

Department of Radiology, University Medical Center Utrecht, Utrecht, The Netherlands

R. van Hillegersberg

Department of Surgery, University Medical Center Utrecht, Utrecht, The Netherlands together were compared for assessing the diagnostic value of EUS.

Results In total, 211 patients (155 men; mean age of $64 \pm 9.4$ years) were included, of which $176(83 \%)$ underwent all three staging investigations. Based on preoperative staging, $173(82 \%)$ patients were considered resectable and $38(18 \%)$ nonresectable. Of all 173 initially resectable patients, 145 were operated on. Of these patients, five $(3.4 \%)$ tumors were found nonresectable during surgery. Postoperative sensitivity, specificity, PPV, and NPV of $\mathrm{CT} / \mathrm{US}$ and CT/US + EUS for predicting surgical resectability were 88 versus $87 \%, 20$ versus $40 \%, 97$ versus $98 \%$, and 6 versus $10 \%$, respectively.

Conclusions Although EUS adds to the specificity of preoperative esophageal cancer staging after $\mathrm{CT}$ chest and abdomen, and US of the neck have been performed, the overall added value of EUS is limited.

Keywords Esophageal cancer - Endoscopic ultrasonography $\cdot$ Surgical resectability

Approximately $50 \%$ of patients with esophageal cancer present with potentially resectable disease [1]. Current guidelines recommend computed tomography (CT) of the chest and abdomen, ultrasonography (US) of the neck, and endoscopic ultrasonography (EUS) for staging of esophageal cancer [2-4]. Although CT of the chest and abdomen and US of the neck are primarily used for the detection of distant metastases, EUS provides information on local resectability (T stage) and locoregional lymphadenopathy (N stage) $[5,6]$.

One of the main goals of esophageal cancer staging is to select patients who are suitable for surgical resection of the tumor, because surgery remains the best curative option for 
patients without distant metastases or tumor ingrowth in adjacent structures outside the diaphragm or pleura. Although recent meta-analyses have shown that EUS has a high sensitivity (75-84 \%) and specificity (65-75\%) for T and $\mathrm{N}$ staging $[5,6]$, the impact of EUS findings for final surgical decision making (resectable or nonresectable) after CT chest and abdomen and US neck have been performed, is largely unknown. Moreover, well-designed comparative impact studies are not available and published data on this topic are conflicting [7, 8]. Other drawbacks of EUS include its invasive character and the risk of esophageal perforation during the procedure [9].

The goal of this study was to evaluate the diagnostic value of staging EUS for determining surgical resectability of esophageal cancer after CT chest and abdomen and US neck have been performed and how often the outcome of staging EUS would have changed the therapeutic management of these patients.

\section{Patients and methods}

All consecutive patients with biopsy-proven esophageal cancer who underwent staging EUS between June 2006 and May 2010 at the Department of Gastroenterology and Hepatology of the University Medical Center Utrecht, the Netherlands, a tertiary referral center for treatment of esophageal cancer, were included. The patient search was performed in the electronic endoscopy report system Endoalpha Documentation (Olympus, Hamburg, Germany) using the key words "EUS" and "staging esophageal/ gastric cardia cancer." All patients underwent a standardized preoperative staging protocol, consisting of a CT chest and abdomen, US neck, and EUS esophagus and upper abdomen. All data were collected from the medical charts.

\section{Staging investigations}

\section{CT chest and abdomen}

A 16- or 64-multidetector row spiral CT scanner (Philips MX 8000 IDT and Philips Brilliance, respectively; Philips Medical Systems, Best, The Netherlands or Somatom Sensation; Siemens Medical Systems, Erlangen, Germany) was used. Thoracic and upper abdomen CT scans were performed after oral contrast intake and intravenous contrast injection, using a detector configuration of $16 \mathrm{~mm}$ or $64 \times 0.75 \mathrm{~mm}$, $120 \mathrm{kVp}$, individualized mAs, a tube rotation time of $0.5 \mathrm{~s}$, and a pitch of 1.3. Overlapping transverse sections were reconstructed using a slice thickness of $5 \mathrm{~mm}$ at $4-\mathrm{mm}$ increments. CT images were evaluated for the presence of the primary tumor, invasion into adjacent structures, presence of locoregional lymph nodes (N0-1), and distant organ metastases (M0-1), according TNM-classification version 6. Tumors were judged as T4 stage or tumor stage less than T4. Lymph nodes measuring $10 \mathrm{~mm}$ or more were considered tumor positive. For patients who underwent multiple CT scans, the latest scan was included in the analysis because the final decision on resectability was based on this scan.

\section{US neck}

Cervical lymph nodes with a diameter of $6 \mathrm{~mm}$ or more were considered to be potentially malignant and subsequent fine needle aspiration (FNA) was performed. In addition, lymph nodes were punctured for FNA if their structure or shape was suggestive of malignancy, i.e., round, hypoechoic with sharp margins. The FNA result was defined as positive if cytology confirmed malignancy and negative if no suspected lymph nodes were reported on US or if FNA did not yield malignant cells.

\section{EUS}

All EUS examinations were performed by a single endoscopist $(\mathrm{FV})$, who has performed $>350$ EUS procedures over the years, using a radial scanning echoendoscope (GFUM130 or GF-UE160, Olympus, Hamburg, Germany). Patients received sedation with midazolam and pethidine or fentanyl. Tumors were staged (TNM) according to the 6th edition of the UICC, which was used in the study period [10]. Dilation of the tumor was not performed if the echoendoscope could not pass the tumor.

\section{Management after tumor staging}

When CT, US, and EUS in a patient with esophageal cancer had been performed, resectability of the tumor was discussed in the multidisciplinary gastrointestinal oncology team, consisting of a surgeon, radiologist, radiotherapist, pathologist, medical oncologist, and gastroenterologist. Additional examinations, such as PET, MRI, or bronchoscopy, were only performed when there was doubt of resectability based on the outcomes of CT, US, and/or EUS. If no evidence of distant metastases or invasion into adjacent organs was found except for ingrowth in the pleura or diaphragm, tumors were considered resectable. Patients who were fit to undergo surgery underwent esophagectomy with or without neoadjuvant treatment (initial chemotherapy for adenocarcinoma alone, later chemoradiation for squamous cell carcinoma, and chemotherapy for adenocarcinoma). Preoperative, nonresectable disease was defined as the composite of either (1) proven metastases by biopsy or mortality within 6 months after diagnosis due to progressive tumor growth, or (2) nonresectability of the tumor as proven by other investigations, such as PET, MRI, or bronchoscopy. 
Postoperatively, nonresectable disease was defined as nonresectability during surgery.

Statistical analysis

Accuracy of thoracic/upper abdominal CT + US neck with or without EUS for predicting surgical resectability were compared to assess the additional value of EUS, by calculating sensitivity, specificity, and positive (PPV) and negative (NPV) predictive value for surgical resectability. This comparison was made preoperatively as well as postoperatively. Preoperatively, the "gold standard" for determining resectability was based on all preoperative staging tests. Postoperatively, the resection itself was the "gold standard." All analyses were performed by using the SPSS version 15.0 (SPSS Inc., Chicago, IL).

\section{Results}

Of the 231 patients who underwent staging EUS, 20 patients were excluded because EUS was the only investigation that had been performed (Fig. 1). This resulted in 211 patients (155 men; mean age of $64 \pm 9.4$ years) that were included. Patient and tumor characteristics are shown in Table 1. Two EUS reports did not provide data on location of the tumor.
Table 1 Patient and tumor characteristics

\begin{tabular}{llr}
\hline Characteristics & $n$ & $\%$ \\
\hline Gender & & 74 \\
Male & 155 & 26 \\
Female & 56 & \\
Mean age (year) & $64( \pm 9.4)$ & 5 \\
Location & & 30 \\
Upper & 10 & 65 \\
Middle & 62 & \\
Lower & 137 & 27 \\
Histologic type & & 71 \\
Squamous & 57 & 2 \\
Adenocarcinoma & 149 & 1 \\
Other & 5 & 25 \\
Histologic grade & & 49 \\
Well differentiated & 1 & 25 \\
Moderate & 53 & 100 \\
Poor & 104 & \\
Undetermined & 53 & \\
Total & 211 & \\
\hline & & \\
\end{tabular}

Of the 211 patients, $176(83 \%)$ underwent all three standard staging investigations. EUS was incomplete in 17 patients due to the presence of a tumor that was stenotic, preventing passage of the echoendoscope. US neck was

Fig. 1 Flowchart

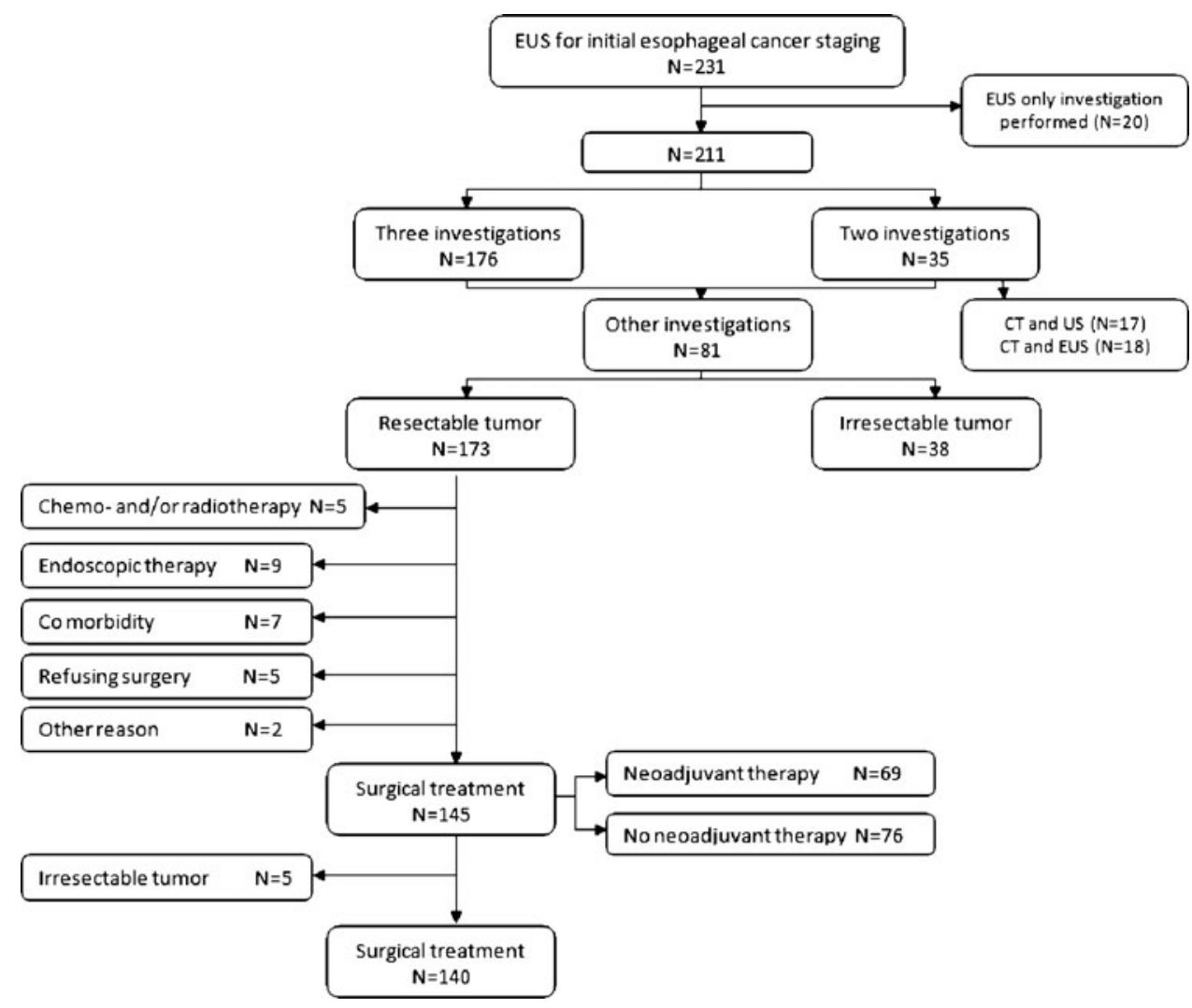


Table 2 TNM stage based on EUS findings

\begin{tabular}{lllllllll}
\hline & $N$ & $\%$ & & $N$ & $\%$ & & $N$ & $\%$ \\
\hline $\mathrm{T} 1$ & 30 & 16 & N0 & 69 & 36 & Mx & 151 & 78 \\
T2 & 13 & 7 & N1 & 123 & 64 & M0 & 13 & 7 \\
T3 & 125 & 65 & Total & 192 & 100 & M1 & & \\
T4 & 23 & & & & & M1a & 24 & 12 \\
T4a & 9 & 5 & & & & M1b & 6 & 3 \\
T4b & 14 & 7 & & & & Total & 194 & 100 \\
Total & 191 & 100 & & & & & & \\
Missing & 20 & & Missing & 19 & & Missing & 17 & \\
\hline
\end{tabular}

missing in 18 patients. In 81 patients, one or more additional investigations were performed, i.e., PET scan (32\%), abdominal ultrasound (25\%), MRI liver (13\%), or bronchoscopy $(13 \%)$. Staging results of EUS, CT, and US neck are shown in Tables 2, 3, and 4. In Tables 5 and 6, the accuracy of EUS for $\mathrm{T}$ and $\mathrm{N}$ staging of patients who did not undergo neoadjuvant therapy is shown, using the pathologic specimen as the "gold standard."

Based on preoperative staging, esophageal cancers were in 173 patients ( $82 \%$ ) considered to be resectable and in 38 $(18 \%)$ nonresectable. Of the latter, 17 patients had biopsyproven metastases (lymph node, $n=10$; kidney, $n=1$; liver, $n=1$; bone, $n=2$; ascites, $n=2$; pleural fluid, $n=1$ ), 9 had tumor progression during neoadjuvant therapy, 2 had biopsy-proven tracheal/bronchial ingrowth, and 10 had convincing evidence of ingrowth or metastases as shown by other investigations (CT, $n=1$; EUS, $n=1$; EUS and CT, $n=3$; CT and PET, $n=1$; EUS and US neck, $n=1$, MRI and PET liver, $n=1$, MRI liver, $n=1$; MRI brain, $n=1$ ). Preoperative sensitivity, specificity, PPV and $\mathrm{NPV}$ of CT/US, and CT/US + EUS for predicting surgical resectability were 88 versus $87 \%, 76$ versus $87 \%, 94$ versus $97 \%$, and 58 versus $59 \%$, respectively (Table 7).

Of all 173 patients who were considered to be resectable after preoperative staging, 145 (84\%) underwent surgery. In 14 patients another treatment modality was performed (endoscopic mucosal resection or definitive chemoradiation), 5 patients refused surgery, 7 were not operable due to severe comorbidity, and 2 had other reasons. Of the 145 operated patients, 69 patients $(49 \%)$ had received neoadjuvant therapy before surgery.

Five $(3.4 \%)$ patients were found to have a nonresectable tumor during surgery. Three of them had ingrowth in adjacent structures (pulmonary vein and right main bronchus, $n=1$; right main bronchus, $n=1$; aorta and celiac trunk, $n=1$ ) and two patients had metastases that had not been detected during preoperative staging (liver metastasis, $n=1$; retroperitoneal metastasis, $n=1$ ). In two of these patients, complete EUS had not been performed because the tumor was too stenotic and passage of the echoendoscope was not possible. Two of these five patients had received neoadjuvant chemotherapy. Postoperative sensitivities, specificities, PPVs, and NPVs of CT/US and CT/ US + EUS for predicting surgical resectability were 88 versus $87 \%, 20$ versus $40 \%, 97$ versus $98 \%$, and 6 versus $10 \%$, respectively (Table 8 ).

Table 3 TNM stage based on CT findings

\begin{tabular}{cllllllll}
\hline & $\mathrm{N}$ & $\%$ & & $\mathrm{~N}$ & $\%$ & & $\mathrm{~N}$ & $\%$ \\
\hline $\mathrm{T}<4$ & 196 & 93 & $\mathrm{~N} 0$ & 108 & 51 & $\mathrm{cM} 0$ & 177 & 84 \\
$\mathrm{~T} 4 \mathrm{a}$ & 3 & 1 & $\mathrm{~N} 1$ & 103 & 49 & $\mathrm{cM} 1$ & 33 & 15 \\
$\mathrm{~T} 4 \mathrm{~b}$ & 12 & 6 & & & & $\mathrm{pM} 1$ & 1 & 1 \\
Total & 211 & 100 & Total & 211 & 100 & Total & 211 & 100 \\
\hline
\end{tabular}

Table 4 TNM stage based on US of the neck results

\begin{tabular}{lrr}
\hline & $N$ & $\%$ \\
\hline cM0 & 178 & 92 \\
cM1 & 1 & 1 \\
pM1 & 14 & 7 \\
Total & 193 & 100 \\
Missing & 18 &
\end{tabular}

Table 5 Accuracy of EUS for tumor (T) depth staging of patients without neoadjuvant therapy

\begin{tabular}{|c|c|c|c|c|c|c|c|c|}
\hline \multirow[t]{2}{*}{ EUS $T$ stage } & \multicolumn{8}{|c|}{ Pathologic $\mathrm{T}$ stage } \\
\hline & $\mathrm{T} 1$ & $\mathrm{~T} 2$ & $\mathrm{~T} 3$ & $\mathrm{~T} 4$ & T0 & Tis & Total & $\%$ correct \\
\hline $\mathrm{T} 1$ & 14 & 0 & 0 & 0 & 0 & 1 & 15 & 93 \\
\hline $\mathrm{T} 2$ & 3 & 1 & 0 & 0 & 0 & 0 & 4 & 25 \\
\hline T3 & 1 & 2 & 37 & 2 & 0 & 0 & 42 & 88 \\
\hline $\mathrm{T} 4$ & 0 & 0 & 3 & 4 & 0 & 0 & 7 & 57 \\
\hline Total & 18 & 3 & 40 & 6 & 0 & 1 & 68 & 84 \\
\hline Overstaged & $22 \%$ & $67 \%$ & $8 \%$ & & & & & \\
\hline Understaged & & & & $33 \%$ & & & & \\
\hline
\end{tabular}

The number of cases in which EUS and pathology results were equal are depicted in bold 
Table 6 Accuracy of EUS for lymph node (N) staging of patients without neoadjuvant therapy

\begin{tabular}{lllll}
\hline EUS & \multicolumn{4}{l}{ Pathological stage } \\
\cline { 2 - 5 } & N0 & N1 & Total & $\%$ correct \\
\hline N0 & $\mathbf{1 4}$ & 12 & 26 & 64 \\
N1 & 6 & $\mathbf{3 7}$ & 43 & 86 \\
Total & 20 & 49 & $\mathbf{6 9}$ & 74 \\
Overstaged & $30 \%$ & & & \\
Understaged & & $25 \%$ & & \\
\hline
\end{tabular}

The number of cases in which EUS and pathology results were equal are depicted in bold

Table 7 Preoperative sensitivity, specificity, PPV, and NPV of CT + US and CT + US + EUS for predicting surgical resectability

\begin{tabular}{|c|c|c|c|c|}
\hline & \multicolumn{2}{|c|}{ Resectable } & Tonresectable & Total \\
\hline \multicolumn{5}{|l|}{$\mathrm{CT}+\mathrm{US}$} \\
\hline Resectable & \multicolumn{2}{|l|}{152} & 9 & 161 \\
\hline Nonresectable & \multicolumn{2}{|l|}{21} & 29 & 50 \\
\hline \multicolumn{5}{|l|}{$\mathrm{CT}+\mathrm{US}+\mathrm{EUS}$} \\
\hline Resectable & \multicolumn{2}{|l|}{150} & 5 & 155 \\
\hline Nonresectable & \multicolumn{2}{|l|}{23} & 33 & 56 \\
\hline \multirow[t]{2}{*}{ Total } & 173 & \multicolumn{2}{|c|}{38} & 211 \\
\hline & PPV (\%) & NPV (\%) & $\begin{array}{l}\text { Sensitivity } \\
(\%)\end{array}$ & $\begin{array}{l}\text { Specificity } \\
(\%)\end{array}$ \\
\hline $\mathrm{CT}+\mathrm{US}$ & 94 & 58 & 88 & 76 \\
\hline $\mathrm{CT}+\mathrm{US}+\mathrm{EUS}$ & 97 & 59 & 87 & 87 \\
\hline
\end{tabular}

Table 8 Postoperative sensitivity, specificity, PPV, and NPV of $\mathrm{CT}+\mathrm{US}$ and CT + US + EUS for predicting surgical resectability

\begin{tabular}{|c|c|c|c|c|}
\hline & \multicolumn{2}{|c|}{ Resectable } & Nonresectable & Total \\
\hline \multicolumn{5}{|l|}{$\mathrm{CT}+\mathrm{US}$} \\
\hline Resectable & \multicolumn{2}{|l|}{123} & 4 & 127 \\
\hline Nonresectable & \multicolumn{2}{|l|}{17} & 1 & 18 \\
\hline \multicolumn{5}{|l|}{$\mathrm{CT}+\mathrm{US}+\mathrm{EUS}$} \\
\hline Resectable & \multicolumn{2}{|l|}{122} & 3 & 125 \\
\hline Nonresectable & \multicolumn{2}{|l|}{18} & 2 & 20 \\
\hline \multirow[t]{2}{*}{ Total } & \multicolumn{2}{|l|}{140} & 5 & 145 \\
\hline & $\begin{array}{l}\text { PPV } \\
(\%)\end{array}$ & $\begin{array}{l}\text { NPV } \\
(\%)\end{array}$ & $\begin{array}{l}\text { Sensitivity } \\
(\%)\end{array}$ & $\begin{array}{l}\text { Specificity } \\
(\%)\end{array}$ \\
\hline $\mathrm{CT}+\mathrm{US}$ & 97 & 6 & 88 & 20 \\
\hline $\mathrm{CT}+\mathrm{US}+\mathrm{EUS}$ & 98 & 10 & 87 & 40 \\
\hline
\end{tabular}

\section{Discussion}

In this study, we show that the diagnostic value of staging EUS for determining surgical resectability when staging with thoracic/abdominal CT and US neck performed in the setting of our institution was limited. The PPV for determining resectability increased nonsignificantly from 97 to $98 \%$; in other words, only in 1 of 100 cases the addition of EUS to thoracic and abdominal CT and US neck resulted in a clinically significant result, i.e., a surgical resection was not performed. The specificity for determining nonresectability increased from 20 to $40 \%$ by adding EUS; however, this outcome is based on data of only 5 of 211 patients (Table 8).

Avoiding unnecessary surgery for nonresectable esophageal cancer is one of the main objectives of preoperative staging with EUS. It is widely accepted that EUS has the highest accuracy for $\mathrm{T}$ and $\mathrm{N}$ staging of esophageal cancer [5]. EUS is regarded as superior to CT in this regard and is thought to be more accurate for evaluation of resectability [11-15]. However, randomized trials comparing the clinical impact of staging protocols with and without EUS have not been performed. A recently published prospective cohort study also showed that EUS was insensitive with respect to resectability and the authors concluded that it should not be performed upfront in every patient [16]. Furthermore, the impact of a diagnostic finding of EUS on treatment policy often depends on the locally used treatment protocol [2]. In the Netherlands, the vast majority of esophageal cancer patients who are staged T2-3N01M0-1a (UICC edition 6) are treated by neoadjuvant chemo(radio)therapy followed by esophagectomy. Because patients with a T2NOM0 tumor are treated similarly as patients with a T3N1M1a tumor, the impact on treatment of differentiating between T2 or T3 tumors by EUS is very low or even absent. Moreover, it has recently been shown that EUS in T1 lesions has a low accuracy for distinguishing submucosal (T1sm) from mucosal cancer (T1m). Recently, Pouw et al. [17] reported that EUS had virtually no clinical impact on the workup of early esophageal neoplasia (T1N0). A diagnostic endoscopic mucosal resection (EMR), which may be therapeutic if the lesion is confined to the mucosal layer is the preferred intervention for staging of these early lesions. Finally, only 14 of 211 tumors $(6.6 \%)$ were considered nonresectable on EUS because they were staged as T4b (UICC TNM 7th edition). Based on this, we recommend performing thoracic/ abdominal CT and US neck upfront, followed by EUS if its result may affect further treatment, i.e., as despite the result of CT doubt on resectability remains (Fig. 2).

Our study focused on the additional value of EUS for determining surgical resectability. However, EUS also is able to address other clinical endpoints, such as pretreatment TNM stage, overall clinical management, and survival, which may be important factor for predicting a patient's prognosis. A reduced survival has been shown to be associated with higher numbers of affected lymph nodes, a factor that has now been incorporated in the 


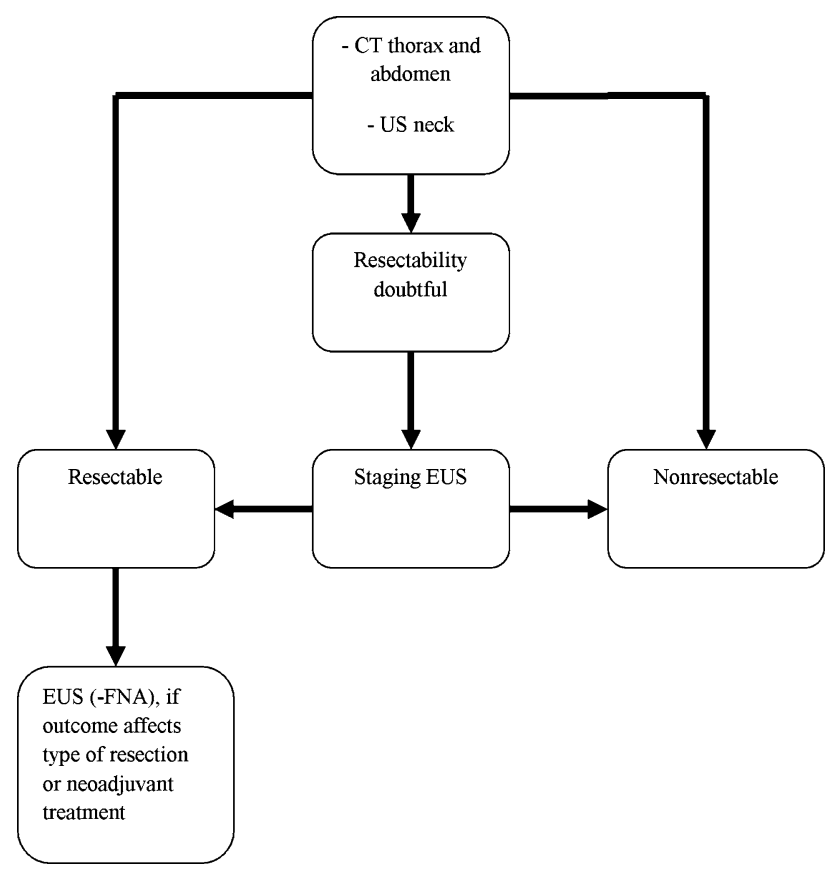

Fig. 2 Flowchart of proposed staging protocol for patients with esophageal cancer

newest version (7th) of the TNM-classification [18]. Moreover, the surgical approach for esophagectomy, i.e., transhiatal or transthoracic, may depend on the presence and determination of the exact location of malignant lymph nodes. Malignant mediastinal lymph nodes located along the distal esophagus can be resected effectively via the transhiatal route. In contrast, malignant supracranial nodes should be removed by a transthoracic approach. EUS, combined with FNA for cytology, has a high accuracy for determining the benign or malignant nature of a lymph node [5, 19, 20], even in low-volume EUS centers [21]. However, the strongest prognostic factor and determinant for a therapeutic option remains the presence of distant metastases, for which CT thorax and abdomen and US neck are the most reliable techniques for detection.

The choice for a transhiatal or transthoracic surgical approach not only depends on nodal involvement, but also is determined by tumor location, i.e., the Siewert classification [22]. The Dutch esophageal cancer guideline recommends performing a radical transthoracic resection with abdominal and mediastinal lymph node dissection in case of Siewert type-I tumors (adenocarcinoma of the distal esophagus). Transhiatal esophagectomy with resection of distal periesophageal and peri-truncal nodes and nodes along the left gastric artery is advised in patients with Siewert type II-III tumors (cardia carcinoma-subcardiac gastric cancer). Total gastrectomy instead of esophagectomy may be an alternative surgical approach to Siewert type II-III tumors [2]. EUS combined with endoscopy may assist in determining the Siewert classification, although data on this are scarce [23].
Pedrazzani et al. [23] showed that the sensitivity and specificity for preoperative staging of Siewert type I cancers with a combination of endoscopy and EUS were 90 and $82 \%$, respectively, whereas these scores decreased to 75 and $69 \%$, respectively, when only endoscopy was performed. Distinguishing type II from type III lesions seemed even more difficult with sensitivities well below $75 \%$. However, the number of patients included in this study was relatively small, therefore, these data should be interpreted with caution.

A potential limitation of our study may be its retrospective design, but because all consecutive patients in a given time interval were included, no bias in patient selection occurred. Another drawback may be the nature of our institution, which is a referral center for the surgical and endoscopic treatment of esophageal cancer. In the majority of patients, the staging workup, mostly US neck and abdominal/thoracic CT, had already been performed in the referring hospital. Most patients who are undergoing staging EUS in our clinic have been referred for surgery or EMR, consequently patients with metastasized or overtly nonresectable disease are rarely seen for EUS. In our opinion, this unlikely reduces the strength of our findings. In contrast, inclusion of the above-mentioned patient category will even further reduce the impact of EUS on determining surgical resectability. We deliberately did not exclude patients in whom passage of the echoendoscope was not possible, as this is an inherent limitation of EUS.

\section{Conclusions}

Although EUS adds to the specificity of esophageal cancer staging after abdominal/thoracic CT and US neck have been performed, the added value for surgical decision making seems limited in our setting. We therefore think that EUS should not be a standard examination in the diagnostic workup of all patients with esophageal cancer but rather should be performed if the outcome likely affects further treatment. More studies are needed to determine in detail in what subgroup of patients with esophageal cancer EUS adds to the treatment decision.

Disclosures M. van Zoonen, M.G.H. van Oijen, M.S. van Leeuwen, R. van Hillegersberg, P.D. Siersema, and F.P. Vleggaar have no conflicts of interest or financial ties to disclose.

Open Access This article is distributed under the terms of the Creative Commons Attribution License which permits any use, distribution, and reproduction in any medium, provided the original author(s) and the source are credited.

\section{References}

1. Enzinger PC, Mayer RJ (2003) Esophageal cancer. N Engl J Med 349:2241-2252 
2. Dutch guidelines esophageal cancer (revised 2010). www.onco line.nl

3. Wang KK, Wongkeesong M, Buttar NS (2005) American Gastroenterological Association Medical Position Statement: role of the gastroenterologist in the management of esophageal cancer. Gastroenterology 128:1468-1470

4. Lightdale CJ (1999) Esophageal cancer. Am J Gastroenterol 94: 20-29

5. Puli SR, Reddy JB, Bechtold ML, Antillon D, Ibdah JA, Antillon MR (2008) Staging accuracy of esophageal cancer by endoscopic ultrasound: a meta-analysis and systematic review. World J Gastroenterol 14:1479-1490

6. Van Vliet EP, Heijenbrok-Kal MH, Hunink MG, Kuipers EJ, Siersema PD (2008) Staging investigations for oesophageal cancer: a meta-analysis. Br J Cancer 98:547-557

7. Pfau PR, Perlman SB, Stanko P, Frick TJ, Gopal DV, Said A et al (2007) The role and clinical value of EUS in a multimodality esophageal carcinoma staging program with CT and positron emission tomography. Gastrointest Endosc 65:377-384

8. Mortensen MB, Edwin B, Hunerbein M, Liedman B, Nielsen HO, Hovendal C (2007) Impact of endoscopic ultrasonography (EUS) on surgical decision-making in upper gastrointestinal tract cancer: an international multicenter study. Surg Endosc 21:431-438

9. Mortensen MB, Fristrup C, Holm FS, PLess T, Durup J, Ainsworth AP et al (2005) Prospective evaluation of patient tolerability, satisfaction with patient information, and complications in endoscopic ultrasonography. Endoscopy 37:146-153

10. Sobin LH, Wittekind C (2002) International union against cancer (UICC) TNM classification of malignant tumours, 6th edn. Wiley, New York

11. Eloubeidi MA, Wallace MB, Reed CE, Hadzijahic N, Lewin DN, van Velse A et al (2001) The utility of EUS and EUS-guided fine needle aspiration in detecting celiac lymph node metastasis in patients with esophageal cancer: a single-center experience. Gastrointest Endosc 54:714-719

12. Rasanen JV, Sihvo EI, Knuuti MJ, Minn HR, Luostarinen ME, Laippala P et al (2003) Prospective analysis of accuracy of positron emission tomography, computed tomography, and endoscopic ultrasonography in staging of adenocarcinoma of the esophagus and the esophagogastric junction. Ann Surg Oncol 10:954-960

13. Romagnuolo J, Scott J, Hawes RH, Hoffman BJ, Reed CE, Aithal GP et al (2002) Helical CT versus EUS with fine needle aspiration for celiac nodal assessment in patients with esophageal cancer. Gastrointest Endosc 55:648-654

14. Wallace MB, Nietert PJ, Earle C, Krasna MJ, Hawes RH, Hoffman BJ et al (2002) An analysis of multiple staging management strategies for carcinoma of the esophagus: computed tomography, endoscopic ultrasound, positron emission tomography, and thoracoscopy/laparoscopy. Ann Thorac Surg 74:1026-1032

15. Wakelin SJ, Deans C, Crofts TJ, Allan PL, Plevris JN, PatersonBrown S (2002) A comparison of computerised tomography, laparoscopic ultrasound and endoscopic ultrasound in the preoperative staging of oesophago-gastric carcinoma. Eur J Radiol $41: 161-167$

16. Schreurs LM, Janssens AC, Groen H, Fockens P, van Dullemen HM, van Berge Henegouwen MI et al (2011) Value of EUS in determining curative resectability in reference to CT and FDGPET: the optimal sequence in preoperative staging of esophageal caner? Ann Surg Oncol [Epub ahead of print]

17. Pouw RE, Heldoorn N, Herrero LA, Ten Kate FJ, Visser M, Busch OR et al (2011) Do we still need EUS in the workup of patients with early esophageal neoplasia? A retrospective analysis of 131 cases. Gastrointest Endosc 73:662-668

18. Sobin LH, Gospodarowicz MK, Wittekind C (2010) International Union Against Cancer (UICC) TNM classification of malignant tumours, 7th edn. Wiley, New York

19. Eloubeidi MA, Cerfolio RJ, Bryant AS, Varadarajulu S (2011) Efficacy of endoscopic ultrasound in patients with esophageal cancer predicted to have N0 disease. Eur J Cardiothorac Surg 40(3):636-641

20. Marsman WA, Brink MA, Bergman JJ, Tytgat GN, ten Kate FJ, van Lanschot JJ et al (2006) Potential impact of EUS-FNA staging of proximal lymph nodes in patients with distal esophageal carcinoma. Endoscopy 38:825-829

21. Hirdes MM, Schwartz MP, Tytgat KM, Schlösser NJ, Sie-Go DM, Brink MA et al (2010) Performance of EUS-FNA for mediastinal lymphadenopathy: impact on patient management and costs in low-volume EUS centers. Surg Endosc 24:22602267

22. Siewert JR, Stein HJ (1998) Classification of carcinoma of the oesophagogastric junction. Br J Surg 85:1457-1459

23. Pedrazzani C, Bernini M, Giacopuzzi S, Pugliese R, Catalano F, Festini $M$ et al (2005) Evaluation of Siewert classification in gastro-esophageal junction adenocarcinoma: what is the role of endoscopic ultrasonography? J Surg Oncol 91:226-231 